\title{
Una Experiencia de Terapia de grupo con niños en edad de latencia.
}

One experience of therapy group with latency age children.

Ignacio Vidal a, Ignacio Vera ${ }^{\mathrm{b}}$, Consuelo Escudero ${ }^{\mathrm{c}}$.

${ }^{a}$ Psiquiatra. Centro de Salud Mental de Getafe, España. ${ }^{b}$ Psiquiatra. Centro de Salud Mental de Getafe, España. ${ }^{c}$ Psicólogo Clínico. Centro de Salud Mental de Getafe, España.

Correspondencia: Ignacio Vidal Navarro (nachete117@hotmail.com)

Recibido: 28/02/2012; aceptado: 12/11/2012

RESUMEN: El presente trabajo describe nuestra experiencia clínica del trabajo terapéutico con un grupo de niños en edad de latencia, con la técnica de juego, realizado en nuestro centro público durante un curso escolar.

PALABRAS CLAVE: latencia, psicoterapia de grupo, juego.
ABSTRACT: The present work describe our clinic experience with a group of children in the latency period, working with play technique in context of group therapy during a school year. KEY WORDS: latency period, group therapy, play.

\section{Latencia}

Se denomina latencia en literatura psicoanalítica al período comprendido entre la declinación de la sexualidad infantil (quinto o sexto año) y el comienzo de la pubertad (undécimo o duodécimo). Durante él se observa, desde este punto de vista, una disminución de las actividades sexuales, la desexualización de las relaciones de objeto y de los sentimientos (especialmente el predominio de la ternura sobre los deseos sexuales) y la aparición de sentimientos como el pudor y el asco, así como de aspiraciones morales y estéticas. Según Freud (1), el período de latencia tiene su origen en la declinación del complejo de Edipo; corresponde a una intensificación de la represión (que provoca una amnesia que abarca los primeros años), una transformación de las catexis de objetos en identificaciones con los padres y un desarrollo de las sublimaciones. Dicho de otro modo, la salida del conflicto edípico y la represión de las pulsiones que han estado activas en la elaboración del conflicto permite su utilización al servicio de la socialización, las relaciones paritarias y el aprendizaje, con la consiguiente apertura hacia el mundo externo a la familia. 
La petición de ayuda en salud mental de niños y adolescentes y la importancia de los grupos en los dispositivos terapéuticos

Probablemente en cualquier Programa de Salud Mental Ambulatorio de Niños y Adolescentes podemos encontrar con mucha frecuencia derivaciones de niños en este período etario con sintomatología en el registro neurótico (2), que podrían designarse como síntomas o signos de "fallo" o "desviaciones" en la latencia (inquietud psicomotriz y trastornos de conducta, dificultades de aprendizaje, falta de atención, fobias, nerviosismo y ansiedad, impulsividad, enuresis...). Los integrantes del grupo que se va a comentar a continuación podrían ser una muestra muy representativa de las demandas a nuestro dispositivo ambulatorio.

A la hora de realizar una planificación clínico-terapéutica de cada caso en el programa de niños y adolescentes, debemos tener en cuenta cuatro factores fundamentales: factor biológico, factor psicológico-psicoterapéutico, factor social y factor familiar (3).

Centrándonos en el punto de vista psicológico, normalidad y patología requieren un planteamiento complejo. Reconocido el síntoma, se considerará su importancia y su función dinámica, y se intentará situarlo en su contexto estructural. Así, la estructura psicopatológica se valora dentro del marco del estudio de los factores biológicos y del medio ambiente que rodea al niño. Desarrollo dinámico, maduración, son, por sí mismos, fuentes de conflicto, y como conflictos, generadores potenciales de síntomas. La multicausalidad estudiada por la psicopatología evolutiva remite a la idea de "niño en su contexto evolutivo", a la idea de la interacción entre elementos biológicos, psicógenos, familiares y sociales, tanto en la estructuración del niño sano como en la génesis de la patología mental.

Nos encontramos principalmente con dos tipos de problemas cuando nos disponemos a realizar psicoterapia con niños: 1) al no ser ellos mismos los que solicitan la ayuda es muy probable que se encuentren poco motivados, además de no identificar como "problemas" o "síntomas" las conductas referidas por sus cuidadores. Los sufrimientos en los niños muchas veces llevan a poner en marcha defensas de carácter, que cuando están suficientemente integradas en el Yo, ya no son sentidas como extrañas y los niños consideren que el sufrimiento proviene del exterior; 2) los niños es probable que tengan dificultades para involucrarse en una conversación sobre su vida, en pasado, con un adulto desconocido, por una limitada capacidad de auto-observación y una inmadurez de las capacidades de síntesis e integración. Además la terapia con niños ha de tener en cuenta la aparición de reacciones transferenciales y contratransferenciales específicas y la necesidad de buscar formas de comunicación adecuadas a la edad y grado de desarrollo simbólico y lingüístico del niño. 
ORIGINALES Y REVISIONES

Burszjten (4) menciona algunas de las dificultades para la psicoterapia individual de los latentes. Señala que el latente proyecta con mucha mayor dificultad su mundo fantasmático sobre el material de juego o de dibujo en la relación analítica, pues durante este período se constata ahora "un vuelco en el régimen de intercambios con los adultos": mientras que durante el período edípico (3-6 años), los padres, así como los demás adultos, eran objetos susceptibles de satisfacer las pulsiones y los demás niños eran vividos como rivales, durante la latencia, son los padres los que aparecen cada vez más como prohibidores de las satisfacciones pulsionales (adquisición del superyó), mientras que los niños de su edad comienzan a ser investidos de forma positiva.

La relación con un adulto, especialmente en una situación dual, es vivida como una invitación peligrosa a volver a un sistema de investimiento anterior, o incluso como un intento de "seducción". De ahí que no nos extrañe que el niño haga todo lo posible para reconducir la situación analítica, a priori extraña, hacia la banalidad de una relación pedagógica. De entrada, el terapeuta le resulta a menudo un personaje peligroso: una especie de juez inquietante y persecutorio a quien va a tener que "contarle todo", o una especie de profesor o pedagogo con el que tiene problemas desde hace tiempo. Pero y sobre todo, es posible que viva esta propuesta de un nuevo interlocutor para sus demandas y quejas que él quisiera dirigirlas a sus propios padres, como un "escaqueo" y una amenaza.

Los latentes se sienten más involucrados en las situaciones actuales (el conflicto está activo) y los miedos y frustraciones ligados a los problemas de integración (las relaciones con los iguales). Consideramos, así pues, que los grupos terapéuticos son una herramienta muy útil para el cambio en niños por estas razones y por otras respecto a la gestión de intervenciones en un dispositivo público (referentes a las ventajas que conllevan los grupos respecto a las consultas individuales respecto al coste-beneficio de la intervención).

\section{Antecedentes de la terapia grupal con niños}

Hace ya varios años que se trabaja en grupos terapéuticos con niños. Slavson (5) a partir de los años 40 del siglo XX, inicia el abordaje de trastornos psiquiátricos en niños y adolescentes dentro de un encuadre psicoterapéutico grupal. Este autor concibe el grupo de actividad como un agregado de individuos que se tratan juntos, pero que son investigados separadamente; en ningún momento son una entidad o un todo, así pues, los esclarecimientos en intervenciones que se realizan son únicamente individuales para este autor. Se trata de crear un ambiente permisivo y de apoyo, y procurar el máximo de intercambio entre pares. Slavson consideraba que 
el despliegue de la dinámica transferencial en el grupo era de la siguiente forma: el grupo era la madre, el terapeuta el padre y los compañeros los hermanos.

A partir de la década de los 50, Anzieu aporta como novedad el intento de unir el psicodrama, el psicoanálisis y los grupos de niños y adolescentes. Este autor plantea la transferencia y la asociación libre (planteada en el grupo como una invitación a ser escuchados) con posibilidad de desplegarse en el espacio (un espacio donde la palabra está privilegiada). El grupo se irá configurando a medida que avancen las sesiones y se vaya conformando una red de identificaciones cruzadas, que irán constituyendo la matriz de identificación grupal. Se introduce el encuadre psicoanalítico grupal, donde se establecen las pautas de funcionamiento del grupo, respecto al tiempo, el espacio y el secreto (donde nada de lo que se comente en el grupo puede ser dicho fuera), con el objetivo de crear un espacio confiable.

Ya en la década de los 60, Foulkes y Anthony amplían el enfoque con la introducción del refuerzo en análisis del grupo y no del individuo, siguiendo los aportes de Bion (la idea de un proceso inconsciente colectivo al cual contribuye cada individuo y que une a los miembros). Estos autores consideran al grupo como totalidad y como el principal instrumento terapéutico y centran la actividad en la interpretación de las defensas de manera verbal.

Más recientemente, Vanni (6) en Italia y Privat (7) en Francia, que trabajan principalmente con niños en período de latencia, remarcan la utilidad del juego como instrumento terapéutico, siguiendo las aportaciones de Winnicott en este campo. Señalan que los temas que más a menudo se plantean en la dinámica del grupo de latentes son los relativos a la dependencia-independencia, la competitividad y control de la agresividad, la diferenciación sexual, la capacidad para cambiar de rol y la revisión de las teorías infantiles sobre la familia.

\section{El proceso terapéutico grupal}

Corresponde a los terapeutas, fundadores del grupo, el primer día de sesión grupal introducir el marco terapéutico y enunciar el encuadre. El primer día, tras la presentación y un breve resumen de los motivos por los que se encuentran todos allí, se enuncia la tarea del grupo ("trabajar en los problemas de cada uno"), el modo de hacerlo ("con la palabra y/o el juego" "diciendo o jugando a lo que a uno se le ocurra") y la necesidad de cuidar y cuidarse para poder trabajar en el grupo : ("no se puede pegar ni insultar, no se puede pintar ni romper las paredes, no se puede salir de la habitación durante los 75 minutos de la sesión, y existe un compromiso para acudir al grupo, no se puede faltar al grupo a menos que se esté enfermo" "no se comenta fuera del grupo lo que ocurre dentro de éste"). 
ORIGINALES Y REVISIONES

En el período inicial el grupo es percibido como algo inquietante en su organización, los niños tienden a funcionar como en el contexto educativo (pasividad y espera del liderazgo por parte del adulto) por ser el que tienen de referencia a esta edad. Los terapeutas, adultos inhabitualmente no directivos, les desconciertan, suscitan mucha inquietud en relación con los límites y los riesgos de desbordamientos. Si pueden decir todo lo que se le pase por la cabeza (asociación libre) aparece el temor a volverse loco y romperlo todo, que les lleva a verificar la solidez del marco físico. Esto despierta el miedo a lo desconocido y por proyección, a lo desconocido de cada uno. Los primeros días de encuentros grupales, los niños se muestran inhibidos en un principio, y pasan con rapidez a la descarga psicomotriz y al descontrol (se quitan los juguetes de las manos, tienen interacciones muy breves entre ellos y buscan en principio interaccionar de forma individual con el adulto). Estas conductas son una prueba de la seguridad y consistencia de los límites y del encuadre.

Al comienzo los psicoterapeutas participan de los intercambios, siendo su función más importante, favorecer lo más posible las interrelaciones entre los niños, que un principio tienden a dirigirse a él. Así, los niños quieren establecer un contacto privilegiado con el/los terapeutas, es decir, cada uno trata de excluir al resto, negar el grupo y lo que tiene de peligroso. Por ello, el hecho de que el terapeuta remita al grupo las preguntas y oriente a los niños a una tarea común favorece la investidura grupal, apareciendo progresivamente un intento de organización que en cierta manera sería el inicio de la búsqueda de una identidad común. Anzieu lo llama "ilusión grupal": investidura del grupo como portador de gratificaciones narcisistas, pudiendo actuar el grupo como coterapeuta. A través de los movimientos transferenciales de cada uno sobre el grupo, se ha constituido una historia común (un mito), y basándonos en esta historia ahora las transferencias pueden ser interpretadas, y en este momento ya se pueden hacer interpretaciones individualizadas a cada uno de los miembros.

A lo largo del proceso de constitución del grupo se observan tentativas de distanciamiento de los objetos perseguidores, de buscar un chivo expiatorio entre los miembros del grupo, como mecanismo para preservar la unidad grupal, haciendo necesaria la proyección del objeto malo destructor hacia fuera (esto se plasma también en los juegos, los niños juegan a que uno de ellos es encerrado en un calabozo construido con cojines y todo el resto del grupo lo aplasta durante 30 segundos, y se van intercambiando...) Siguiendo con esto suele ocurrir que las proyección de las partes malas recae sobre la figura del terapeuta, que se convierte entonces en el excluido del grupo (no le dejan hablar, le dan la espalda, no le escuchan...se puede pensar como una analogía del asesinato simbólico del padre enunciado por Freud, que permite que el grupo se estructure en la fraternidad...).

El terapeuta, contratransferencialmente, debe reconocer un sentimiento de impotencia infantil para evitar contraactuaciones, y demostrando su comprensión 
del mundo de la infancia va a propiciar un verdadero encuentro identificatorio. También es importante que sepa seguir siendo el garante del marco terapéutico y que mantenga este papel, consistente en estar a la vez en el grupo y fuera del grupo, como la persona que puede conferirle un sentido.

Glasserman y Sirlin (8) ponen énfasis en la interpretación como parte natural de la conversación, que va apenas más allá de lo que están expresando los niños con palabras o con el juego. También remarcan que tan importante es interpretar como saber oír y participar en lo que se oye, así estimula a todos el aprendizaje de escucharse e intentar comprenderse, llevándolos con el tiempo al pensamiento reflexivo. Enuncian un concepto muy interesante: el encuadre interno del terapeuta respecto al grupo, para explicar que el terapeuta debe tener claro qué espera que suceda y dentro de qué tipo de relaciones y actividades va a desenvolverse el proceso terapéutico (de ahí la importancia de la formación teórica y práctica del profesional).

Naturalmente la dinámica del grupo no es un recorrido lineal, aparecerán de forma recurrente los períodos de ansiedad, sobre todo en momentos de separación por vacaciones, exámenes o cuando se produce alguna otra circunstancia desfavorable en la vida familiar de cada niño.

Rosa Jaitin (9), que trabaja con niños en instituciones de crianza infantil, pone énfasis en la empatía como instrumento terapéutico. Según Jaitin, la empatía posibilita aliviar el sufrimiento que produce la carencia. El sujeto que es criado con ternura (acción maternante que provee alimento, calor, mecimiento y que inscribe al ser humano como sujeto cultural) puede ubicarse con facilidad en el lugar del otro, porque también sabe registrar sus carencias y los modos para sentirse ligado y satisfecho. El reconocer lo propio (autoconocimiento) posibilita ubicarse más fácilmente en la necesidad del otro.

\section{El juego en el grupo de latentes}

El juego es una parte fundamental del desarrollo de los niños. Los niños juegan para aliviar tensiones, explorar la realidad, aprender y relacionarse con otros, tanto adultos como pares. Winnicott plantea que el juego se desarrolla en una zona intermedia que abarca elementos subjetivos y lo se percibe del exterior (espacio transicional) (10). Esta interacción produce un estado de ensimismamiento comparable a la concentración del adulto. Asimismo, el juego sigue la ley de la asociación libre y remite a la historia personal y familiar de cada niño (11). Los niños establecen diferentes tipos de juegos en función de con quien se juega, es decir de la relación que se establece con los adultos y con los iguales. Si los niños juegan 
ORIGINALES Y REVISIONES

con un adulto desarrollan juegos según el modelo adulto-niño, en una relación vertical, actuando papeles o realizando actividades que saben que el adulto quiere, pero que ellos no harían con otros niños. Los juguetes se utilizan de una manera formal, cumpliendo el objetivo para el que se han fabricado (a las muñecas se les da de comer, se les viste, con los coches se hacen carreras, las verduras de plástico se cocinan...etc.).

Si los niños juegan entre iguales, generalmente sin presencia de los adultos, desarrollan otro tipo de juegos que responde al sistema de relación entre pares. En esta relación horizontal los juegos son completamente diferentes. Se utiliza mucho mas la imaginación, la fantasía, los juguetes pierden su finalidad formal y se pueden utilizar de formas muy diferentes. Este sistema de relación esta fuera de la relación con el adulto pero permanentemente influida por ella.

Las relaciones de los niños con los adultos se organizan sobre el modelo del conflicto edípico y las relaciones paritarias son la sede del aprendizaje por la experiencia. Hay funciones del yo que se desarrollan sobre el modelo adulto y otras por la experiencia del niño en el mundo de los pares. Es un modelo de desarrollo en dos vías diferentes que interactúan una sobre la otra y ambas necesarias.

El juego, visto desde la dinámica grupal, facilita el pasaje desde la "cultura de pares" a la "cultura del grupo terapéutico", siguiendo la línea de Bión. La "cultura de pares" responde a las referencias internas que los niños traen de su experiencia en otros grupos. Fundamentalmente la familia y el colegio. En estos contextos los adultos siempre ostenta una autoridad definida, son los que imponen las normas, aplican castigos si se incumplen y en general intervienen para resolver conflictos, enseñar, dar consignas o proteger y cuidar al que lo necesita. Por tanto los niños esperan en el comienzo del grupo que el terapeuta adulto cumpla también estas funciones.

"La cultura del grupo terapéutico" pasa por la aceptación de los afectos y sentimientos provocados en la interacción grupal. Debido a que muchos se viven como peligrosos y se califican como negativos, tanto por ellos como en general por los adultos, la posibilidad de instrumentalizarlos en el grupo producirá la consiguiente disminución de culpa y temor al castigo. Esta aceptación se realiza fundamentalmente por la experiencia en el juego grupal y se produce gracias a la posibilidad de ver y experimentar, primero en los otros y después en sí mismo, sentimientos rechazados a través del juego y de la acción. Este pasaje se produce progresivamente a medida que el grupo avanza (12). 


\section{Nuestra experiencia}

La forma de trabajo que venimos realizando en nuestro Centro de Salud Mental es próxima a la desarrollada por otros profesionales de la Comunidad de Madrid (13). Los grupos terapéuticos en nuestro centro suman un total de 10 integrantes. Todos ellos tienen entre 8 y 11 años y presentan sintomatología de registro neurótico (trastornos del comportamiento, depresiones, ansiedad, dificultades de las relaciones entre pares, inhibiciones, problemas de rendimiento académico, fobias, enuresis...). Se ha eludido enumerar la codificación diagnóstica de los integrantes por la poca funcionalidad que representa en el presente trabajo (además variaría cada año/grupo siguiente). Los síntomas mencionados se distribuyen ampliamente en nuestra muestra, pues todos los integrantes presentan varios de éstos síntomas/problemas. Los integrantes del grupo se seleccionan de la muestra de derivaciones de ese rango edad a nuestro centro (se excluyen niños con síntomas en el registro psicótico como Trastornos Graves del Espectro Autista, Psicosis Infantiles así como niños con Retraso Mental) Es un grupo cerrado, semanal, que dura aproximadamente 9 meses (temporada de clases del curso escolar), y se trabaja con la técnica de juego. En la sala grupal tienen 10 cojines cúbicos donde pueden sentarse y un baúl con materiales, pinturas, papeles revistas, y diversos juguetes: playmobil, coches, figuras humanas, animales, utensilios de cocina... etc. Los terapeutas son dos, un miembro del staff y un residente (MIR o PIR) que trabajan habitualmente en coterapia.

Los temas que más a menudo se plantean en los grupos de niños en edad de latencia, son los relativos a la dependencia-independencia de los padres y de los adultos en general. El odio, la envidia, el miedo, la venganza, la sumisión, la posesión, el dominio... producidos por la ambivalencia ante la dependencia afectiva, real e imaginaria, de las figuras parentales en particular y de los adultos en general, y simultáneamente, la necesidad de separarse de ellos sin perder el reaseguramiento afectivo, producen conductas agresivas, oposicionistas y de rebeldía que se viven como peligrosas y se califican como negativas.

Debido a la inmadurez y dependencia de los niños, consideramos necesaria la colaboración de los padres para garantizar la continuidad del tratamiento. Se realiza un grupo paralelo de padres, de periodicidad mensual como objetivo de apoyar el tratamiento de los niños y servir de contención a la ansiedad que se moviliza en los padres ante los cambios de sus hijos y para que apoyen los intentos de separaciónindividuación de sus hijos y poder dar sentido a estas conductas "negativas" que implican un esfuerzo de los niños por ser más independientes, además nos sirve de monitorización de los cambios del niño en su ambiente. En ningún caso supone una psicoterapia para los padres.

Además, la experiencia del grupo de padres puede favorecer la revalorización de su función parental, procurándoles un apuntalamiento narcisista y favoreciéndoles una identificación con el sufrimiento de sus hijos. 
ORIGINALES Y REVISIONES

\section{Viñeta clínica}

En las primeras sesiones del grupo aparece el GAME (juegos de acción), como defensa de los niños a la situación grupal. Los niños juegan al futbol-pollo, futbol-cojín, tenis-berenjena...etc. (los niños utilizan los materiales como balones, objetos arrojadizos...sin aprovechar el significado utilitario/simbólico de éstos...). Es el tipo de juego que se da, generalmente, entre los niños en la primeras sesiones grupales y en momentos resistenciales, como juego de descarga psicomotriz sin contenido psíquico.

El PLAY (juego simbólico) se va desarrollando a lo largo de las sesiones, conforme se van complejizando las interacciones entre los pares y cuando el grupo es investido como espacio psicoterapéutico contenedor. Se acondiciona un verdadero espacio transicional en el que puede elaborarse una actividad grupal de pensamiento y de simbolización. Es un juego sin reglas preestablecidas que, como el grupo, va descubriendo sus propias reglas a medida que va desarrollándose, y se apoya en una creatividad compartida. Los niños intentan jugar todos a alguna cosa, intentan ponerse de acuerdo en cuanto a las reglas y roles de cada uno...

A medida que transcurre el grupo y los niños pueden experimentar todas las posibilidades de roles y conductas con la ayuda de los terapeutas, disminuye la agresión y la ansiedad y aparece la cooperación. Son posible entonces los compromisos y acuerdos, el respeto por las necesidades y debilidades de cada uno y pueden ser capaces de dar y recibir ayuda. Se abre así, la posibilidad de experimentar nuevos roles. Los juegos son más ordenados y menos ruidosos y se empiezan a visualizar algunos cambios. En nuestro grupo, los niños empiezan a organizarse con un juego que consiste en provocarse "terremotos" los unos a los otros, los niños se mantienen tumbados con el vientre sobre el cojín, en equilibrio mientras sus compañeros balancean el cojín provocando "un terremoto". Este juego se organiza en una sesión (cerca de la terminación del grupo) en la que se hablan dificultades que tienen que ver con ser testigos de discusiones y descontroles entre sus padres, los "terremotos" que sienten en su interior cuando ven pelearse a sus padres.

BIBLIOGRAFÍA:

(1) Laplanche J, Pontalis JB. Diccionario de Psicoanálisis. Buenos Aires. Paidós. 2007

(2) Espín JC, Belloso JJ. Estudio descriptivo de los niños y adolescentes nuevos vistos en un servicio de salud mental en el período de un año. Rev. Asoc. Esp. Neuropsiq., 2009, vol. XXIX, n. ${ }^{\circ}$ 104, pp. 329-354.

(3) Gutiérrez JR, Rey F (Eds.) Planificación terapéutica de los trastornos psiquiátricos de niños y adolescentes. Madrid. Smith-Kline-Beecham. 2000 
(4) Bursztejn C. Psicoterapia en el período de latencia. Cuadernos de psiquiatría y psicoterapia del niño y del adolescente. $\mathrm{N}^{\circ} 26$. Pags 73-86. 1998

(5) Gamo E, Gómez R (Coord.). Grupos Terapéuticos y Asistencia Pública. Madrid. A.E.N. Estudios. 1997

(6) Vanni F. Un modelo grupal en la psicoterapia infantil. Revista Clínica y Análisis grupal, $1990, \mathrm{n}^{\circ} 55$, pp. 353-355

(7) Privat P, Quelin-Soulinox D. El niño en psicoterapia de grupo. Paris. Editorial Síntesis. 2000

(8) Glasserman MR, Sirlin ME. Psicoterapia de grupo en niños. Buenos Aires. Ediciones Nueva Visión. 1979

(9) Jaitin R. Apoyos grupales en la crianza infantil. Buenos Aires. Cinco.1987

(10) Winnicot DW. Realidad y juego. Barcelona. Gedisa. 1971

(11) Gamo E (Coord.). Ante la mirada de los otros: guía de terapias grupales. Madrid. Editorial Plaza. 2007

(12) Escudero, C. Psicoterapia de grupo con niños en edad de latencia. Revista Norte de Salud Mental. $\mathrm{N}^{\circ} 24.2005$

(13) Groch JE, De la Iglesia L, Villanueva C. Actualización en Psicoterapia de Grupo de orientación Psicodinámica con Niños y Adolescentes en los Servicios de Salud Mental de la Comunidad de Madrid. Cuadernos de Psiquiatría y Psicoterapia del Niño y Adolescente. № 29. Pags 33-40. 2000 